\title{
Philosophiques
}

\section{Les premières articulations du fonctionnement intentionnel : le projet d'un Raumbuch chez Husserl entre 1892 et 1894}

\section{Robert Brisart}

Volume 34, numéro 2, automne 2007

URI : https://id.erudit.org/iderudit/016988ar

DOI : https://doi.org/10.7202/016988ar

Aller au sommaire du numéro

Éditeur(s)

Société de philosophie du Québec

ISSN

0316-2923 (imprimé)

1492-1391 (numérique)

Découvrir la revue

Citer cet article

Brisart, R. (2007). Les premières articulations du fonctionnement intentionnel : le projet d'un Raumbuch chez Husserl entre 1892 et 1894. Philosophiques, 34(2), 259-272. https://doi.org/10.7202/016988ar d'utilisation que vous pouvez consulter en ligne. 


\title{
Les premières articulations du fonctionnement intentionnel: le projet d'un Raumbuch chez Husserl entre 1892 et 1894
}

\author{
ROBERT BRISART \\ Facultés universitaires Saint-Louis à Bruxelles
}

Grâce à l'édition réalisée par Strohmeyer et publiée dans la seconde partie du volume XXI des Husserliana, on sait qu'en 1892 Husserl entreprit plusieurs séries de recherches consacrées à la philosophie de la géométrie qui l'amenèrent, dès octobre 1893, à nourrir le projet d'un ouvrage sur l'espace dont très vite l'ampleur de la problématique lui parut telle qu'il entama aussi, dès cette date, un journal consacré à la programmation et à l'état d'avancement de ce qui devait donc devenir un Raumbuch, lequel néanmoins ne verra jamais le jour. Pourquoi donc, au lendemain de la Philosophie de l'arithmétique, alors que, comme annoncé, il était censé en rédiger le second tome, Husserl dévia-t-il ses réflexions vers la géométrie et s'attaqua plus exactement au problème de l'origine de la notion formelle d'espace ? Sans aucun doute, la réponse à cette question exige-t-elle de libérer les quelques textes de Husserl préparatoires au Raumbuch du cadre par trop étroit de questions seulement relatives à la pure théorie de la géométrie où les a confinés Strohmeyer dans l'introduction au demeurant richement documentée de son édition. À l'inverse, il nous semble que, pour qu'en soient mieux perçus les enjeux, ces textes qui, pour l'essentiel, traitent autant de l'espace de l'intuition que de l'espace de la géométrie, afin précisément d'en élucider le rapport, gagneraient à être rattachés à l'ensemble des manuscrits de recherches en psychologie des années 1893-1894 et que viendra ponctuer l'article sur les Études psychologiques pour la logique élémentaire, lesquels ont tous été édités dans le volume XXII des Husserliana. Ainsi s'agirait-il de montrer que la réponse à notre question liminaire peut éclairer l'itinéraire qui, de sa première œuvre, conduisit progressivement Husserl au dégagement et à l'articulation de la double vie intentionnelle de la conscience, d'une part, celle de l'intuitivité, et, d'autre part, celle de l'apophanticité. Ce travail de longue haleine entrepris donc de très bonne heure n'a pas seulement fait qu'initier l'une des tâches séminales de la phénoménologie des Recherches logiques de 1901, mais il a inauguré aussi tout le programme de l'œuvre à venir, tant il vrai qu'en filigrane de la théorie de la connaissance sans cesse redéployée par Husserl jusqu'au soir de sa vie, il n'a jamais été question d'autre chose que de réécrire une esthétique et une logique transcendantales pour cerner au plus près les relations généalogiques et téléologiques qu'entretiennent entre elles les deux modalités intuitives et significationnelles de l'intentionnalité. 
Bien qu'il n'en soit que très indirectement question dans sa Philosophie de l'arithmétique ${ }^{1}$, Husserl ne s'est pas pour autant désintéressé de la géométrie et donc de la question de l'espace à l'époque de sa première publication. Au semestre d'hiver 1889-1890, il fit en effet un cours intitulé "Questions choisies de philosophie des mathématiques » dont une longue partie fut, à la demande de ses étudiants, consacrée à un " Aperçu historique sur le développement de la géométrie ", et dont le texte est reproduit dans le même volume XXI des Husserliana ${ }^{2}$. Dans ce cours, Husserl, qui manifestement connaît de très près tous les linéaments du développement récent de la géométrie, rappelle les trois périodes qui ont marqué l'histoire de l'axiome XI des Éléments d'Euclide, dit encore le cinquième postulat, soit l'axiome des parallèles qui, dans sa version traditionnelle, demande que si deux lignes droites sont coupées par une troisième de telle manière que les angles intérieurs situés d'un même côté ont une somme inférieure à deux droits, elles se rencontrent du côté considéré quand on les prolonge suffisamment.

La première période fut d'abord celle où l'on pensa que cette proposition était démontrable a priori, c'est-à-dire qu'elle était nécessairement déductible des concepts qu'elle contient. Néanmoins, parce qu'en sa formulation même le cinquième postulat ressemblait davantage à un théorème plutôt qu'à un axiome ${ }^{3}$, il apparut tout aussi vite qu'il ne pouvait avoir le caractère d'une proposition immédiatement analytique, mais paraissait plutôt devoir être déductible des autres postulats d'Euclide. Et c'est d'ailleurs à cette démonstration que, depuis l'Antiquité, s'étaient employées des générations de mathématiciens, mais sans succès, au point que d'Alembert lui-même n'hésitera pas à considérer l'irrésolution du problème posé par le postulat des parallèles comme «le véritable scandale des Éléments de géométrie " ${ }^{4}$. L'objection demeurait donc que si ce postulat était intuitivement compréhensible, il lui manquait d'être logiquement évident et qu'en tout cas sa dérivation à partir d'éléments fondés sur l'intuition ou l'imagination ne pouvait suppléer aux exigences de sa dérivation purement logique à partir des autres axiomes.

La seconde période de l'histoire du cinquième postulat fut essentiellement marquée par les travaux de Lobatchevski, de Bolyai et de Gauss, qui, bien loin d'apporter la démonstration depuis si longtemps attendue, menaient au contraire, selon les propres dires de ce dernier, à rendre douteuse la vérité de la géométrie euclidienne traditionnelle. Gauss, par exemple, montrait tout d'abord l'indépendance du postulat des parallèles pour établir ensuite que l'on pouvait très bien s'en passer ou, comme dans la géométrie imaginaire de Lobatchevski, choisir un postulat contradictoire et le combiner avec les autres

1. Cf. Hua XII, p. 32 sq à propos de la synthèse spatiale de Lange et Baumann. Philosophie de l'arithmétique, trad. fr. J. English, Paris, 1972, p. 43 sq. (cité PA dans la suite).

2. Hua XXI, pp. 312-347.

3. Cf. Hua XXI, p. 318.

4. Cf. Hua XXI, p. 320. 
axiomes d'Euclide pour déduire de nombreux théorèmes en contradiction avec les propositions euclidiennes. La preuve commençait ainsi d'être faite qu'une géométrie non euclidienne pouvait constituer une alternative logique à la géométrie euclidienne classique et qu'en outre, selon Gauss, elle pouvait tout aussi bien servir à décrire l'espace comme si celui-ci ne devait pas être forcément euclidien.

Mais, comme le rappelle Husserl dans ses leçons de 1889-1890, ce n'est qu'au cours d'une troisième période essentiellement marquée par les travaux de Riemann-Helmholtz que l'idée d'une géométrie non euclidienne va considérablement s'élargir jusqu'au point de se présenter désormais non plus seulement comme une alternative à la géométrie euclidienne, et tout aussi cohérente qu'elle, mais comme une théorie unifiée du plan et de l'espace à l'intérieur de laquelle la conception euclidienne n'apparaîtra plus que comme un cas particulier de multiplicité parmi d'autres. Le seul ressort de ce changement considérable fut d'abandonner l'ancienne méthode axiomatique pour procéder à une arithmétisation du plan et de l'espace grâce à laquelle leur géométrie devenait déterminable en fonction seulement de la mesure de leur courbure.

Ainsi, en matière de planimétrie à laquelle Husserl consacre un développement important dans ses leçons, trois cas deviennent envisageables parmi lesquels le plan euclidien bidimensionnel avec toutes ses propriétés classiques n'apparaît plus désormais que comme le cas extrêmement particulier d'un plan dont la courbure est de constante égale à 0 . Dans un tel cas, le point le plus court entre deux points est toujours effectivement une droite par rapport à laquelle ne passe qu'une et une seule parallèle par un point extérieur à elle. Mais si l'on considère que la courbure du même plan peut être aussi de constante positive, nous obtenons alors une géométrie elliptique de ce plan dont il découle que les lignes de la plus courte trajectoire se coupent dans tous les cas et qu'il n'existe donc pas de parallèles en ce plan. Alors que si la courbure du plan est de constante négative, nous en obtenons enfin une géométrie hyperbolique où par un point donné par rapport à une droite fixée il devient possible de tracer un faisceau de parallèles. Tous ces résultats déduits de façon purement analytique montrent donc que la planimétrie euclidienne ne représente, en fonction de la courbure du plan, qu'un niveau de calcul parmi d'autres laissant apparaître la polymorphie de multiplicités à géométries variables. En outre, il est bien évident, remarque Husserl, que si l'on considère l'espace comme "l'arrière-fond en lequel sont contenues les surfaces en tant que figures et si nous le comparons à ces figures, alors apparaissent des analogies caractéristiques $»^{5}$. Autrement dit, nous pouvons nous doter d'une expression analytique qui permet de parler d'une mesure de courbure de l'espace et considérer ainsi l'espace comme une multiplicité tridimensionnelle de mesure de courbure nulle, c'est-à-dire comme une multiplicité de type euclidien. Mais que nous ayons précisément exprimé comme égale à 0 la mesure 
de la courbure d'une telle multiplicité en fait aussitôt un cas particulier parmi d'autres déterminations analytiques de mesure de courbures constantes et, partant, de mesure de courbures variables. Si l'on tente dès lors de traduire la notion d'espace à partir de la généralisation de toutes les différentes façons de le déterminer analytiquement en fonction de la mesure de sa courbure, on peut parler «de multiplicités à $n$ dimensions dont les éléments ou les points sont déterminés par $n$ coordonnées ${ }^{6}$.

Ce qui de toute évidence dut retenir l'attention de Husserl dans ce développement récent de la géométrie, et plus encore dans la réévaluation des géométries non euclidiennes faite par Riemann, c'est sans aucun doute l'introduction du concept de multiplicité pour traiter géométriquement de l'espace. Lorsque, à l'extrême fin de ses leçons de 1889-1890, Husserl dit que, sur le plan purement analytique, la théorie riemannienne de l'espace possède " une valeur durable $»^{7}$ (bleibenden Wert), ce qu'il maintiendra notamment dans une lettre à Brentano du 29-12-1892 où il répète qu'elle n'est pas sans avoir « un contenu plein de valeur ${ }^{8}$ (Wertvolle Gehalt), on devine assez facilement même s'il n'en dit pas grand chose - que cette valeur tient essentiellement au mode de traitement riemannien des multiplicités avec lesquelles justement Husserl se débat à l'époque dans le cadre qui lui est propre, à savoir celui d'une réflexion sur les multiplicités en arithmétique et surtout sur la logique de leur extension dans le but de fournir une détermination rigoureuse à tout le champ de l'arithmetica universalis. À cet égard, toute l'inventivité de Riemann, qui ne pouvait qu'intéresser Husserl dans ses propres recherches en arithmétique, tient essentiellement à sa Mannigfaltigkeitslehre, laquelle consiste tout d'abord à montrer qu'à l'inverse d'un ensemble, une multiplicité ne détermine pas les éléments qu'elle rassemble en fonction de leur matière, c'est-à-dire des propriétés qui leur sont communes, mais qu'elle détermine seulement ses éléments par des formes déterminées de connexion purement analytiques dont l'expression est algébrique. En outre, ce que Riemann a également indiqué, et qui ne pouvait qu'apparaître comme du plus haut intérêt à Husserl alors confronté au problème de l'extension arithmétique, c'est que le passage d'un genre de multiplicité à un autre très différent est obtenu lui aussi de façon purement formelle moyennant, par exemple, l'expression analytique des variations du degré de courbure de l'espace ou du plan en géométrie.

Outre le fait de libérer ainsi le concept géométrique d'espace de tout recours intuitif à la spatialité, l'arithmétisation de la géométrie, dont Riemann fut l'un des pionniers, eut pour conséquence directe de rendre les objets ou plus précisément les champs d'objets géométriques totalement corrélatifs à des procédures d'engendrement réglées par des opérations déterminées. En d'autres mots, les champs d'objets, c'est-à-dire les multiplicités, pouvaient dès

6. Hua XXI, p. 333.

7. Hua XXI, p. 347.

8. Hua Dok III, 1, p. 11. 
lors apparaître comme autant de régions purement formelles seulement déterminées par des règles analytiques. Il en découlait encore qu'un espace spécifiquement géométrique n'était plus qu'une multiplicité dont la dimension perdait toute signification spatiale pour ne garder que la signification algébrique d'un certain nombre de variables ou de signes numériques sur lesquels les opérations ne pouvaient plus être assimilées à des constructions spatiales, mais seulement à des fonctions algorithmiques. Nul doute qu'aux yeux de Husserl cette arithmétisation de la géométrie non seulement revenait à en faire un bon échantillon de l'arithmetica universalis, mais de surcroît pouvait aussi servir d'étalon pour déterminer ce à quoi elle devait dès lors ressembler. Ce que reconnaîtra très explicitement Husserl, notamment dans une lettre à Carl Stumpf datée de février 1890, en la définissant comme "un système de signes et d'opérations sur ces signes " ${ }^{9}$, c'est-à-dire, ajoutera-t-il, "en harmonie avec Helmholtz » ${ }^{10}$, sous-entendu donc aussi de façon assez proche du formalisme analytique de Riemann lui-même.

S'il y a donc aux yeux de Husserl quelques bonnes raisons pour reconnaître une valeur mathématique à la géométrie de Riemann, il n'en reste pas moins que l'autre conclusion du cours de 1889-1890 est que d'un point de vue philosophique elle reste "sans valeur ${ }^{11}$. Autrement dit, comme le répétera Husserl dans la même lettre à Brentano de décembre 1892 où il reconnaissait à la géométrie de Riemann/Helmholtz un "contenu plein de valeur », le défaut qui leur reste néanmoins imputable est de «n'être parvenus à la moindre clarté philosophique sur les fondements de leur science ${ }^{12} »$. On ne s'étonnera pas de l'ambivalence manifeste de l'appréciation de Husserl, puisqu'elle est en quelque sorte indexée sur la propre ambivalence de la Philosophie de l'arithmétique eu égard à laquelle doit être bien sûr recontextualisée l'approche husserlienne de la géométrie entre 1890 et 1892.

On le sait, le premier ouvrage de Husserl est marqué d'une rupture interne qui effectivement finit par l'orienter vers une conception purement formaliste de l'arithmétique. Étant d'abord parti de l'idée, qui le dirigeait encore dans son Habilitationsschrift de 1887, selon laquelle c'est le concept de numération qui constitue le fondement de l'arithmétique, Husserl fait volte-face au chapitre X de l'ouvrage pour remarquer que, si l'arithmétique est essentiellement faite d'opérations sur des nombres, alors il ne se peut que ceux-ci soient pris au sens propre, c'est-à-dire comme des concepts pour des quantités dont nous serions dès lors chaque fois contraints d'avoir une représentation. Ce sont donc les opérations et, avec elles, l'extension de l'arithmétique qui amènent Husserl à reconsidérer le matériau sur lequel elle procède en admettant désormais qu'il ne s'agit que de signes dont nous n'avons qu'une représentation impropre, tant

9. Hua XXI, p. 246.

10. Hua XXI, p. 247.

11. Hua XXI, p. 347.

12. Hua Dok III, 1, p. 11. 
il y a impropriété à parler en ce cas d'une représentation, c'est-à-dire d'une intuition quelconque.

Les conséquences de cette bifurcation sont au moins triples. Tout d'abord, du fait que l'arithmétique relève désormais d'un processus exclusivement signitif, la compréhension de la formation d'un système de nombres semble donc ne requérir plus rien d'autre qu'une sémiotique destinée à montrer comment l'arithmétique tout entière se constitue par algorithmes, c'est-à-dire par procédures purement techniques de calcul permettant la production de certaines catégories de nombres à l'intérieur de certaines chaînes opératoires. Ensuite, du fait que l'extension des nombres et, avec elle, le développement progressif de l'arithmetica universalis paraissent désormais ne résulter que de l'extension des techniques algorithmiques engendrant de nouvelles formations de signes chaque fois formellement dérivées des formations plus élémentaires, comme l'écrit Husserl à Stumpf dans la lettre de 1890 déjà citée, l'arithmetica universalis peut dès lors être comprise "non pas comme une science, mais comme une partie de la logique formelle, qu'[il] définir[a] [lui]-même comme un art des signes ${ }^{13}{ }^{\prime}$. Troisièmement, enfin, la critique du formalisme qui était au départ adressée notamment à Helmholtz tant que Husserl considérait que le fondement de l'arithmétique ramenait au concept de numération devient désormais sans objet, puisque le recours aux processus signitifs revient à adopter une position formaliste et nominaliste qui notamment transparaît très clairement dans cet extrait de 1889-1890:

Le concept de numération n'autorise aucune extension du tout; ce qui est en extension et autorise l'extension, c'est seulement la technique arithmétique. C'est elle qui connaît et elle qui forme les signes négatifs, imaginaires, irrationnels et fractionnaires qui servent à rendre le calcul plus complet et qui de ce point de vue ont une signification logique importante. Mais ce qui leur manque, c'est tout contenu conceptuel par delà l'algorithmique ${ }^{14}$.

Toutefois l'ambivalence que nous pointions à propos de la Philosophie de l'arithmétique ne consiste pas en ce revirement en faveur du signe et au détriment du concept ou de toute signification par delà la technique arithmétique. Elle tient bien davantage au fait que la Philosophie de l'arithmétique ne s'épuise pas dans la sémiotique de l'arithmetica universalis, ni donc n'en reste à son formalisme, mais que, en tant que philosophie justement, elle ne renonce pas à cette question que l'on aurait pu croire définitivement abandonnée et que pourtant Husserl pose ouvertement au chapitre XI de l'ouvrage : «sur quoi se fonde et s'appuie la symbolisation $?^{15}$ ". Si l'invention husserlienne de la phénoménologie a quelque part une origine, c'est bel et bien ici. En voici l'idée : pour être complète une théorie de la science, et quelle que soit la science en

13. Hua XXI, p. 248.

14. Hua XXI, pp. 42-43.

15. Hua XII, p. 220 ; PA, p. 241. 
question, ne saurait se clore sur elle-même par une exposition de ses concepts fondamentaux ou de ses principes. De ceux-ci, il reste encore à avérer l'évidence que nous en avons par réflexion sur les actes où ces concepts et ces principes trouvent un étayage ou un fondement. En l'occurrence, si les représentations signitives ou symboliques échouent à procurer l'objet en une donation primaire, et c'est bien en ce sens qu'elles sont des représentations impropres, elles n'en sont pas moins des représentations objectivantes dans la mesure où, par la médiation du signe, c'est bien un objet et, en arithmétique, plus précisément une quantité ou une multiplicité dénombrable qui est signifiée et, par là, visée à vide. La question proprement philosophique est dès lors de savoir comment nous pouvons nous rapporter à quelque chose comme une multiplicité, d'abord la plus simple, c'est-à-dire matérielle ou sensible, pour poser sur cette base la question de la modalité selon laquelle peuvent nous être données des multiplicités formelles comme celles auxquelles nous avons affaire en arithmétique. Ce travail spécifiquement philosophique est, comme le dit encore Husserl dans la Philosophe de l'arithmétique, "l'étude de fond de la formation et de la signification des représentations symboliques sur le domaine du nombre ${ }^{16}$ ".

Il ne nous importe pas ici d'analyser la façon dont Husserl a entrepris ce travail en 1890, mais, pour en revenir à notre propos, d'avancer que c'est assurément l'absence d'un tel travail dans la théorie géométrique de RiemannHelmholtz, ou du moins sa confusion avec un travail de construction d'un tout autre genre, qui justifie qu'à la même époque Husserl dénie à cette théorie la moindre valeur philosophique. Dans le cours de 1889-1890, la critique adressée à Riemann au nom même de la philosophie, mais qui, dans le chef de Husserl, s'étend aussi aux théories de Gauss et de Helmholtz, revient à leur contester le bien-fondé qu'il y a à prétendre fonder la géométrie sur une théorie de la courbure. Les recherches de Riemann, rappelle Husserl, visent à fournir au moyen d'une théorie générale les signes caractéristiques par lesquels une multiplicité plane se distingue d'une multiplicité courbe. Ce qui suffit à concevoir les multiplicités planes comme un cas tout à fait particulier parmi une infinité de cas possibles, puisque la planéité renvoie à une valeur déterminée, parmi d'autres, de la mesure de la courbe, celle précisément où cette valeur est égale à 0 . Contre quoi Husserl fait valoir que n'importe quelle valeur de courbure d'un plan ou d'un espace n'est déterminable que relativement à la planéité du plan ou de l'espace. Autrement dit, il existe une priorité logique de la multiplicité plane par rapport à toute multiplicité courbe et par conséquent une priorité logique aussi de la géométrie euclidienne par rapport aux géométries non euclidiennes.

C'est une erreur de croire, écrit Husserl, pouvoir déduire le concept de multiplicité plane à partir de celui de multiplicité courbe. Une telle tentative n'aurait

16. Hua XII, p. 217; PA, p. 238. 
de sens et de signification que si la seconde avait une priorité logique sur la première. Mais ceci est si peu le cas que c'est le concept de multiplicité courbe qui présuppose celui de multiplicité plane ${ }^{17}$.

Ainsi, explique Husserl, les mathématiciens, qui aujourd'hui ne se conforment pas à la priorité logique de la planéité, se comportent comme s’ils voulaient déduire le concept d'unité du concept de nombre sans considérer que, chaque nombre se composant d'unités, chaque concept de nombre présuppose le concept d'unité. C'est exactement le même type de cercle logique que de prétendre fournir le fondement de la géométrie par une théorie de la courbure. Husserl termine enfin le cours de 1889-1890 par la présupposition que «l'on peut effectivement former le concept de multiplicité à $n$ dimensions et que l'on peut donc concevoir des concepts d'espace supérieurs au nôtre. Mais, même de ce point de vue, ajoute-t-il, la recherche de Riemann est lacunaire ${ }^{18}$. N'est-ce pas finalement dans la mesure où ce qui n'est encore qu'une construction, et ne peut être que cela, est pensé à tort par Riemann comme une fondation, se faisant donc passer pour ce qu'elle n'est pas, à savoir une philosophie de la géométrie.

Toutefois, on s'en aperçoit également, la critique de la valeur philosophique de la géométrie riemannienne qui est menée par Husserl en 1890 reste encore, pour ainsi dire, bien en deçà des exigences fondationnelles qui sont celles de la philosophie elle-même. En effet, l'argumentaire philosophique de Husserl contre Riemann s'y appuie uniquement sur la priorité logique de la géométrie euclidienne et donc sur son rôle fondateur par rapport à toute construction de géométries non euclidiennes. Mais qu'est-ce qui, indépendamment de cette priorité logique par rapport à toute construction métagéométrique autorise à penser que le concept de multiplicité tridimensionnelle de type euclidien s'impose en soi comme le concept formel que nous avons prioritairement de l'espace ? Reposée en ces termes, la question fondationnelle de la géométrie ne peut plus se suffire de la logique mais en appelle à une généalogie de cette logique. C'est, peut-on dire, à tenter de réaliser cette tâche d'élucidation philosophique proprement dite que se consacrera Husserl deux ans plus tard, en 1892-1893, dans les analyses menées en vue de son Raumbuch.

Selon la planification établie par Husserl dans le journal qu'il tenait sur l'état d'avancement du Raumbuch ${ }^{19}$, celui-ci avait prévu trois groupes de recherches sur l'espace: psychologiques, logiques et métaphysiques, en donnant manifestement plus de poids au premier groupe, puisque, avec les recherches psychologiques, il y allait de la représentation pré-scientifique de l'espace dont il s'agissait essentiellement de décrire comment c'est de son idéalisation que procède la représentation scientifique en géométrie. Avant donc que d'aborder des recherches logiques notamment consacrées au rapport entre espace euclidien

17. Hua XXI, p. 345.

18. Hua XXI, p. 347.

19. Hua XXI, pp. 404-406. 
et non euclidien, puis des recherches métaphysiques essentiellement consacrées, elles, au rapport entre les multiplicités géométriques et l'espace réel ou effectif, la question était tout d'abord, pour Husserl, d'examiner les divers contenus psychologiques que nous pouvons avoir de l'espace.

Procéder de la sorte, c'est donc bien en venir à la généalogie de la logique géométrique pour tenter de rétablir les liens génétiques existant entre la représentation intuitive et les concepts purement formels qui caractérisent le champ de l'idéalisation scientifique en général. Aussi cette question, qui avait déjà animé les premières recherches de Husserl en arithmétique et dont il avait très vite pressenti le besoin de l'étendre à tout le domaine de la logique dès après sa critique de Schröder en 1891, l'occasion lui était maintenant donnée de la redéployer sur le terrain de la géométrie, c'est-à-dire en ce domaine des mathématiques où le problème prend une acuité exemplaire de savoir comment, à partir de ces contenus abstraits que sont les moments spatiaux de la représentation intuitive pré-scientifique, on passe à cette représentation purement conceptuelle qu'est l'espace géométrique. En somme, la question était de comprendre comment à partir de certains abstracta matériels s'engendrent les abstracta formels de la géométrie : l'espace comme pure multiplicité et ses composants formels tels que points, droites, plans, etc. C'est donc bien la problématique centrale de la Philosophie de l'arithmétique qui est ici remise sur le métier, mais à présent dans le but d'éclairer ce qui ne l'y était que très imparfaitement encore, à savoir les étapes successives que la conscience intentionnelle doit franchir pour qu'à partir des couches les plus élémentaires de la représentation intuitive puissent se former des représentations conceptuelles où se noue le rapport à des objets purement formels.

Exprimée de la sorte, la problématique de fond du Raumbuch - celle en tout cas sur laquelle Husserl a visiblement le plus travaillé en laissant quasiment pour compte les recherches de logique et de métaphysique qui devaient lui faire suite - se rattache clairement, on le voit, au groupe des analyses psychologiques entreprises par ailleurs entre 1892 et 1894, sans que l'on ne voie plus très bien si ces analyses furent menée pour étayer une philosophie de la géométrie ou si celle-ci fut menée pour exemplifier une psychologie essentiellement consacrée à l'articulation de la vie intentionnelle en sa bipolarité, intuitive et conceptuelle. Sans aucun doute est-ce la seconde interprétation qui est la bonne, puisque si le Raumbuch ne verra jamais le jour, c'est notamment en raison de l'étroitesse de sa problématique spécifique par rapport à celle qui, dès 1894, engageait la psychologie husserlienne sur la voie d'une vaste réflexion où se trouvait engagée la question des statuts de l'objet intentionnel. Mais il n'en reste pas moins que l'article publié par Husserl en 1894 sous le titre Études psychologiques pour la logique élémentaire, lequel vient en quelque sorte ponctuer la série d'analyses réalisées par Husserl à cette époque, se clôt en relevant que, si ces analyses ont certes un intérêt purement psychologique, elles ont surtout une importance capitale pour « l'élucidation de l'origine de la représentation de l'espace » où justement « on tombe sur les 
fonctions intuitives et re-présentatives qui ici agissent partout ensemble et sans la compréhension desquelles on tâtonne désemparé dans l'obscurité ${ }^{20}$ ».

Or, disait Husserl dans le premier texte de 1892 préparatoire au Raumbuch, élucider la différence entre représentation intuitive et représentation conceptuelle nécessite d'en revenir de nouveau à la différence entre représentation propre et représentation impropre et aussi à la différence entre représentation abstraite et représentation concrète ${ }^{21}$. Ce sont précisément ces deux couples de questions qui font la substance de l'article de 1894, comme si celui-ci avait été entièrement conçu en réponse à l'une des premières questions prescrites par la philosophie de la géométrie. Cet article est donc fait de deux parties : la première, la plus courte, traite de la distinction entre contenu abstrait et contenu concret de la représentation; la seconde, la plus longue et la plus importante aussi, porte sur "l'intuition » (Anschaunng) et la "représentation» (Repräsentation).

Mais à la détermination de quels paramètres pouvaient donc bien servir les deux axes (concret-abstrait, intuitif-signitif) successivement envisagés en de telles analyses? Pour le voir, contentons-nous ici d'esquisser à grands traits quelques-unes des thèses emblématiques de ces recherches sur l'origine de la géométrie qui bien sûr n'ont eu de sens dans l'ordre des préoccupations de Husserl que dans la mesure où, contrairement à Frege par exemple, jamais il n'a considéré les sources de la géométrie comme seulement intuitives et donc comme étrangères à la logique. Tout au contraire, pour Husserl, la géométrie est une science a priori ${ }^{22}$ dont les fondements sont constitués de concepts purement formels qui possèdent leur propre système de lois formelles, lui-même transposable - ainsi que Hilbert en fera un peu plus tard la preuve dans ses Grundlagen der Geometrie de 1899 — en un ensemble limité et exhaustif de groupes d'axiomes pouvant enfin donner lieu aux multiples inférences logiques ou aux théorèmes de la géométrie tout entière.

Toutefois, le caractère apriorique de la géométrie ne saurait justifier que l'on doive traiter l'espace géométrique de façon purement déductive comme s'il ne relevait que d'une pure construction intellectuelle. Certes, l'espace géométrique est la résultante d'une activité logique, et c'est pourquoi il est un espace seulement pensable et non pas un espace représentable lié donc à l'intuition, ou alors seulement à titre tout à fait occasionnel et inessentiel d'illustration figurative des propositions géométriques ${ }^{23}$. Mais il reste que cette activité logique n'est elle-même qu'une idéalisation conceptuelle de l'expérience intuitive de l'espace ${ }^{24}$. En d'autres mots, ce sont des configurations et des relations

20. Hua XXII, pp. 190-191; Articles sur la logique, trad. fr. J. English, Paris, 1975, pp. $162-163$.

21. Hua XXI, p. 262.

22. Cf. Hua XXI, pp. 296-300.

23. Cf. Hua XXI, p. 271.

24. Hua XXI, p. 286 sq. 
spatiales intuitionnées qui, pour ainsi dire, servent de substrats à l'idéalisation conceptuelle en géométrie.

Or de quelle nature est ce substrat intuitif ? Nous avons l'intuition d'étendues finies, de surfaces déterminées, de lignes qui les séparent en les coupant, et même d'une certaine continuité dans le champ de perception: ce sont là autant de moments abstraits partiels des divers contenus d'intuition. Par modification d'intérêt, l'intuition peut se concentrer sur ces moments abstraits en passant de l'un à l'autre de manière à provoquer un déroulement d'intuitions successives qui d'une portion d'espace finie renvoie à une autre, et ainsi de suite jusqu'à ce que, au bout du compte, soit reconnu l'espace global $(\text { Gesamtraum })^{25}$. Comment cela se produit-il ? Les analyses psychologiques portant sur les déroulements d'intuitions réalisées dans un manuscrit de 1893 intitulé "Intuition et représentation, intention et remplissement ${ }^{26}$ ont précisément servi à le montrer, un contenu primaire ou partiel est chaque fois appréhendé comme renvoyant à un autre qui fait partie de son halo, de telle façon que chaque contenu partiel intuitionné pour lui-même est investi d'une intention qui trouve son remplissement dans le passage à l'intuition d'un autre contenu partiel, et ainsi de suite jusqu'à ce qu'au bout du compte ce soit l'unité objective de la chose qui est reconnue. Pour qu'ait lieu ce genre de déroulement continu d'intuitions, au cours duquel chaque contenu intuitif partiel se double d'une charge signitive renvoyant à un autre contenu partiel selon un processus temporel qui sans cesse fait devenir autre le contenu intuitionné, aucune médiation conceptuelle n'est requise. Par contre cette médiation doit bien l'être pour que soit reconnue l'unité objective vers laquelle est finalement tendu comme vers son but tout le déroulement d'intuitions. Car où cette unité est-elle reconnue explicitement? Non pas dans l'intuition proprement dite, répond Husserl, mais dans les jugements qui l'accompagnent et où, sous une forme conceptuelle, un contenu global se trouve exprimé comme représentant l'unité objective à laquelle sont rattachés, comme autant de ses déterminations, les divers contenus partiels rencontrés dans la suite des intuitions partielles. C'est pourquoi « l'unité objective est une unité par jugement, non pas par simple intuition, mais sur fondement d'intuition ${ }^{27} »$. Telle que reprise dans les textes du Raumbuch, l'idée est donc que la totalité formée par le fusionnement continuel des différentes parties intuitionnées, à savoir ici la totalité spatiale, est une unité objective qui, elle, n'est pas intuitionnée comme telle, mais simplement intentionnée. C’est pourquoi, explique Husserl, il y a lieu de "pratiquer une certaine reservatio mentis lorsque nous parlons de l'espace intuitif », car tel qu'il est reconnu par la conscience commune ou pré-scientifique, "l'espace comme totalité n'est pas intuition, mais déjà une unité

25. Hua XXI, p. 276.

26. Cf. Hua XXII, 275-283; Sur les objets intentionnels, trad. fr. J. English, Paris, 1993, pp. 222-232, (cité OI).

27. Hua XXII, p. 282 ; OI, p. 231. 
logique qui dépend de la représentation conceptuelle et se produit par jugement sur le donné intuitif ${ }^{28}$ ".

Si Husserl maintient donc fermement l'idée d'une fondation de l'espace géométrique dans l'espace de la représentation, elle ne lui semble pas pour autant attenter au caractère a priori ou pur de la géométrie. Cette fondation, en effet, n'est pas directe, mais est stratifiée en plusieurs couches de vécus intentionnels dont la restitution du mode de transformation montre comment la représentation elle-même passe du registre intuitif au registre conceptuel et, de façon corrélative, comment les objets auxquels la conscience a successivement affaire passent du registre matériel au registre idéal et à l'intérieur même de celui-ci au registre purement formel. Derechef, pour l'expliquer, rien ne requiert le recours à des opérations mentales de seconde main au sens classique de l'abstraction, car ces transformations disent elles-mêmes la dynamique ou la vie de la conscience qui, dans le déroulement de son rapport intuitif et originaire aux objets, se voit téléologiquement conduite à sa propre modification en un mode d'exercice intégralement nouveau de l'activité intentionnelle, lequel relève désormais de la représentation par concept ou par signification, dont l'unité idéale, réitérable en une multitude de jugements, traduit le rapport à un autre mode de prestation de l'objet où celui-ci se donne précisément telle une idéalité.

Pour Husserl, c'est ce destin de la conscience que sert à exemplifier la genèse de la géométrie, plus précisément encore dans le cas où l'idéalité objective revêt le caractère du formel pur. En guise d'intuitions de l'espace n'existent en fait que celles qui portent sur les moments spatiaux non autonomes à l'intérieur d'intuitions plus larges, avec cette particularité pourtant que chaque chose possédant son étendue, le moment spatial n'est pas seulement un abstractum matériel, mais aussi un substrat pour toute unité d'intuition, donc un «fondement abstrait ${ }^{29}$ ». Mais tout aussi peu qu'il est une intuition, l'espace en tant que tel, c'est-à-dire comme totalité infinie, n'est pas du même ordre que ses parties finies qui, elles, sont matériellement visibles; il est pour la conscience commune une "figure idéale»(ideelles Gebilde) seulement pensable par représentation conceptuelle ${ }^{30}$. En l'espèce, faudrait-il ajouter, parce que le conceptuel est ici d'une généralité qui ne laisse plus rien filtrer de la matérialité première des moments spatiaux, il exprime le rapport intentionnel de la pensée à une objectivité qui n'est autre qu'une forme générale du domaine des objets matériels en général. Bref, c'est à la représentation pré-scientifique elle-même qu'il appartient de se rapporter à un espace dont l'existence idéale et purement formelle le détache des intuitions premières du spatial. Si, par suite, la géométrie peut se voir conférer le titre de science purement a priori, c'est seulement dans la mesure où elle n'est que "l'élaboration logique ${ }^{31}$ de cette

28. Hua XXI, p. 283.

29. Hua XXI, p. 276.

30. Hua XXI, pp. 283-284.

31. Hua XXI, p. 271. 
objectivité idéale de l'espace dont elle tire ses propres définitions élémentaires et cherche à dégager analytiquement les lois formelles, moyennant l'établissement d'un ensemble exhaustif de groupes d'axiomes.

Concluons. Dans un texte intitulé «Symbole de la géométrie » datant de décembre 1894, c'est-à-dire de l'époque où le projet du Raumbuch semble avoir été abandonné, puisque ce texte fut le dernier que Husserl lui consacra, celui-ci écrit que »un exposé scientifique de la géométrie doit rigoureusement opposer le symbole et la chose en question $($ Sache) ; il doit par des axiomes rigoureux et en se fondant sur eux par un processus purement formel, exclure les erreurs et montrer dans ses limites le processus intuitif ${ }^{32}{ }^{2}$. Écartant ainsi le symbolique de la géométrie, car le symbolique ne traduit jamais que l'intrusion en elle de l'intuition, celle précisément des représentations figuratives qui même là où elles sont possibles ne constituent jamais une preuve formelle, Husserl entend bien accentuer ainsi l'autonomie logique de la géométrie et rappeler, comme il en avait déjà défendu l'idée dans un texte de 1893, qu'elle est une «science purement apriorique ${ }^{33}{ }$. Mais Husserl n'entendait certainement pas remettre pour autant en question que cette logique a une genèse et qu'elle remonte à notre expérience de l'espace. Aussi appartient-il à l'analyse psychologique — à laquelle Husserl ne va pas tarder de préférer le nom de phénoménologie - de pratiquer une eidétique de la conscience d'où puissent être dégagées les transformations successives de l'exercice intentionnel à travers lesquels sont avérées les différentes modalités de la donation objective, depuis le niveau le plus primitif de la réalité matérielle jusqu'au niveau plus élevé des idéalités. Ce travail d'abord entrepris sur le terrain de l'arithmétique, Husserl l'aura bien mieux approfondi encore sur celui de la géométrie, en montrant comment cette science marquée au sceau du pur a priori ne doit son origine qu'à l'abstraction comprise au sens radicalement nouveau d'une réflexion idéalisante sur les actes par lesquels se forment les concepts généraux et où se noue la relation significationnelle à un type d'objectivité regroupant ce que l'on peut appeler les abstracta formels. Mais en raison de cette nécessaire rétro-référence aux actes de significations générales et donc au mode de donation des objets généraux correspondants, les idéalisations de la géométrie,

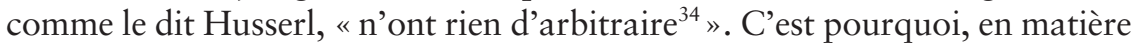
de géométrie, Husserl continue de récuser toute conception purement analytique qui, comme chez Gauss, Helmholtz ou Riemann, ne vise qu'à déterminer de façon métrique n'importe quel espace considéré comme une multiplicité arbitraire à $n$ dimensions. Pour Husserl, au contraire, c'est l'espace tridimensionnel euclidien qui s'impose d'abord à l'idéalisation géométrique, et ce n'est qu'à partir de lui que peuvent être échafaudées ensuite des métagéométries opérant sur des espaces de dimensions supérieures. Par cette restriction fondationnelle

32. Hua XXI, p. 295.

33. Hua XXI, p. 296.

34. Hua XXI, p. 308. 
de la géométrie à l'a priori de l'espace euclidien, Husserl n'entend bien sûr pas s'opposer aux différents développements analytiques qui, à partir de lui, peuvent mener à la construction d'espaces non euclidiens; mais il entend à tout le moins maintenir les liens génétiques par lesquels la géométrie trouve ses conditions de possibilité dans la représentation pré-scientifique de l'espace en comprenant par là non pas tant la représentation intuitive mais, sur la base de son déroulement, la représentation conceptuelle où, dans le développement du fonctionnement intentionnel, l'espace s'impose toujours aux jugements comme tridimensionnel. 